\title{
Gene Ontology Enrichment Analysis of Renal Agenesis: Improving Prenatal Molecular Diagnosis
}

\author{
Silvia Kalantari ${ }^{a, c}$ Isabel Filges ${ }^{a, b}$ \\ ${ }^{a}$ Medical Genetics, Institute of Medical Genetics and Pathology, University Hospital Basel and University of Basel, \\ Basel, Switzerland; bepartment of Clinical Research, University Hospital Basel and University of Basel, Basel, \\ Switzerland; 'Immunogenetics and Transplant Biology Service, Città della Salute e della Scienza University Hospital, \\ Turin, Italy
}

\section{Keywords}

Renal agenesis · Enrichment analysis · Gene ontology ·

Exome sequencing · Prenatal diagnosis

\begin{abstract}
Uni- or bilateral renal agenesis (RA) is a commonly occurring major congenital anomaly impacting fetal and neonatal outcomes. Since the etiology is highly heterogeneous, our aim was to provide a logically structured approach by highlighting the genes in which variants have been identified to be associated with RA and to define the pathways involved in this type of abnormal kidney development. We used Phenolyzer to collect a list of all the genes known as causative for RA. Using ClueGO gene enrichment analysis, we classified the relationship between these genes and the biological processes defined by gene ontology. We identified 287 genes and 69 groups of enriched biological processes. About $50 \%$ included pathways directly related to the development of urogenital organ tissues. Several ciliary, axis specification, hindgut development, and endocrine pathways were enriched, which may relate to different clinical presentations of RA. Our gene ontology enrichment analysis shows that genes representing distinct biological pathways are significantly enriched. This knowledge will lead to an improved molecular diagnosis in clinical care when applying genome-
\end{abstract}

wide sequencing approaches. The findings will also allow to further study the biological pathways involved in RA and to identify novel candidate genes and pathways.

\section{(C) 2021 The Author(s)}

Published by S. Karger AG, Basel

\section{Introduction}

Renal agenesis or aplasia is defined as the absence of one (unilateral renal agenesis) or both kidneys (bilateral renal agenesis). Bilateral renal agenesis (BRA) occurs in $0.1-0.3 / 1,000$ births and is a fatal disorder. Stillbirth and premature birth are both common. Unilateral renal agenesis (URA) has a higher incidence (approximately 1/1,000 births) and a considerably better prognosis, depending, however, on the function of the remaining kidney and the severity of associated anomalies [Talati et al., 2019]. The prevalence of isolated URA is likely higher in adults since it can remain undetected. There is a male preponderance in both unilateral and bilateral agenesis (male:female ratio of 2.5:1) [Harewood et al., 2010]. Renal agenesis, particularly when bilateral, is considered the severe spectrum of congenital anomalies of the kidney and urogenital tract (CAKUT) which account for $20 \%$ of all congenital anomalies.
C 2021 The Author(s).

Published by S. Karger AG, Basel

This is an Open Access article licensed under the Creative Commons Attribution-NonCommercial-4.0 International License (CC BY-NC) (http://www.karger.com/Services/OpenAccessLicense), applicable to the online version of the article only. Usage and distribution for commercial purposes requires written permission.
Correspondence to:

Isabel Filges, isabel.filges@ usb.ch 
Both BRA and URA can occur as an isolated finding, but associated congenital anomalies are common, especially in BRA, and may involve structures in the contiguous developmental field such as ureters, bladder genital organs, anus, and lumbosacral vertebra or may present as a syndromic disorder in up to about $30 \%$ of the cases. When BRA or URA are identified prenatally, it is thus important to examine all other organs and systems for further minor or major anomalies and to observe for neurodevelopmental anomalies later on during childhood [Talati et al., 2019; Bodria and Sanna-Cherchi, 2015].

Due to the advances and availability of prenatal highresolution ultrasound, fetal structural anomalies can be detected earlier in pregnancy, and major organ anomalies diagnosed within the first trimester [Rossi and Prefumo, 2013]. Anhydramnios as a sign of renal agenesis can be present as early as 16-20 gestational weeks [Bianchi et al., 2010]. Once the anomaly is detected, it is of paramount importance to gather as much information as possible about the etiology, prognosis, available treatments, and recurrence risk. The demand to clarify the cause is usually high, but our knowledge concerning the molecular pathogenesis of renal agenesis, particularly when isolated, is still limited, hampering straightforward genetic testing approaches.

Common chromosomal anomalies such as trisomy 13, 18,21 , triploidies and monosomy $\mathrm{X}$ can all present with renal agenesis [Sanna-Cherchi et al., 2007]. Copy number variants (CNVs) account for about $10 \%$ of the congenital kidney abnormalities and for kidney agenesis [Verbitsky et al., 2019]. Therefore, chromosomal microarray (CMA) is usually indicated when renal agenesis is detected. Some recurrent genomic disorders described are microdeletions in 1q21.1, 4p16.1p16.3, 17q12, and 22q11 [Verbitsky et al., 2019]. Table 1 summarizes the monogenic syndromes most commonly associated with renal agenesis. Phenotypic expression, however, may be very variable.

The current hypothesis is that the majority of isolated nonsyndromic renal agenesis is the result of a multifactorial process, where genes are not the only players [Jin et al., 2014]. Exome and genome sequencing studies in patients with CAKUT are providing insights into the genomic landscape by identifying additional susceptibility loci and monogenic causes also for renal agenesis [Groopman et al., 2019; Verbitsky et al., 2019]. Studying the genes, the biological pathways as well as the developmental mechanisms these genes are involved in is mandatory to gain further insight into the complex pathogenesis of renal agenesis.

There are several hypotheses as to the developmental processes which are disturbed in renal agenesis. One hypothesis is that it may be the result of dysplastic kidney regression during fetal development [Mesrobian et al., 1993], referred to as hypodysplasia. Indeed, URA may occur in association with dysplastic alterations in the contralateral kidney. About $11 \%$ of the fetuses bearing lethal bilateral disorders of kidney development at autopsy show co-occurrence of renal agenesis, renal hypoplasia, and dysplastic or cystic dysplastic kidneys [Kleiner et al., 1986]. Therefore, the term renal hypodysplasia/aplasia has been adopted. Currently, there are 3 monogenic disorders described in association with this phenotype (OMIM \#191830, \#615721, and \#617805) caused by pathogenic variants in the ITGA8, FGF20 and GREB1L genes.

Furthermore, renal hypodysplasia and renal agenesis have been hypothesized to result from defects in key steps during nephrogenesis, such as the induction of the ureteric bud from the mesonephric duct or the mesenchymal-epithelial transition. These are complex processes orchestrated by several genes and developmental pathways, whose disruption may lead to the entire spectrum of CAKUT. A molecular monogenic etiology, however, is identified in less than $20 \%$ of the CAKUT cases [Verbitsky et al., 2019], and the same is true for renal agenesis [Wu et al., 2017].

Genome-wide sequencing is used now increasingly often for the clinical diagnosis of such heterogeneous conditions and for the research of novel candidate genes in renal agenesis and CAKUT. Gene lists are extensively used for in- and output of such genomic sequencing, but diagnostic precision and interpretation still is a challenge. We used gene enrichment analysis (EA) as a bioinformatic technique which allows to identify overrepresented and underrepresented functional biological groups within a list of genes [Hall and Kiefer, 2016]. We applied EA to highlight the enriched gene sets in which variants have been identified to be associated with renal agenesis, in order to shed light on the pathways and developmental mechanisms involved. The results can be used for a more structured and meaningful interpretation of genetic causes of renal agenesis in practice, as well as to determine biological pathways so far statistically underrepresented, but holding the potential to identify novel candidate genes and pathways relevant to the etiology. Ultimately, the future development of specific therapies and therapeutic targets will depend on our understanding of such ontological processes.

\section{Materials and Methods}

We used Phenolyzer to generate a list of the genes associated to renal agenesis phenotypes. Phenolyzer is an online tool which can use either disease or phenotype terms to prioritize mendelian and complex disease genes based on the input phenotype description. 
Table 1. Renal agenesis and related monogenic syndromes

\begin{tabular}{|c|c|c|c|}
\hline Syndrome & Genes/CNV & Clinical description & Inheritance \\
\hline Kallman syndrome (OMIM \#308700) & KAL1 & Anosmia, hypogonadotropic hypogonadism, renal agenesis & $\mathrm{XL}$ \\
\hline Goltz-Gorlin syndrome (OMIM \#305600) & PORCN & Dermal hypoplasia, limb defects, cardiac anomalies, renal agenesis & XL \\
\hline $\begin{array}{l}\text { Fraser syndrome } 1 \text { (OMIM \#219000), } 2 \\
\text { (OMIM \#617666), } 3 \text { (OMIM \#617667) }\end{array}$ & $\begin{array}{l}\text { FRAS1, FREM2, GRIP1, } \\
\text { GREM1 }\end{array}$ & $\begin{array}{l}\text { Cryptophthalmos, ambiguous genitalia, cutaneous syndactyly, } \\
\text { malformed ears, dysplastic kidneys, unilateral or bilateral renal } \\
\text { agenesis }\end{array}$ & $\mathrm{AR}$ \\
\hline $\begin{array}{l}\text { Smith-Lemli-Opitz syndrome (OMIM } \\
\# 270400 \text { ) }\end{array}$ & DHCR7 & $\begin{array}{l}\text { 2nd - 3rd toe syndactyly, post-axial polydactyly, growth } \\
\text { restriction, cardiac and brain anomalies, ambiguous genitalia, } \\
\text { ureteropelvic junction obstruction, hypospadias, cystic or } \\
\text { hypoplastic kidneys, unilateral or bilateral renal agenesis }\end{array}$ & $\mathrm{AR}$ \\
\hline $\begin{array}{l}\text { Van Maldergem syndrome } 2 \text { (OMIM } \\
\text { \#615546) }\end{array}$ & FAT4 & $\begin{array}{l}\text { Intellectual disability, typical craniofacial features, auditory } \\
\text { malformations resulting in hearing loss, skeletal and limbs } \\
\text { anomalies, renal hypoplasia and agenesis }\end{array}$ & $\mathrm{AR}$ \\
\hline $\begin{array}{l}\text { Antley-Bixler syndrome (OMIM } \\
\text { \# 207410) }\end{array}$ & FGFR2 & $\begin{array}{l}\text { Bicoronal craniosynostosis (rarely lambdoid and metopic), joint } \\
\text { contractures, choanal atresia, ambiguous genitalia, vertebral } \\
\text { anomalies, thin ribs, renal agenesis }\end{array}$ & $\mathrm{AD}$ \\
\hline $\begin{array}{l}\text { Branchiootorenal syndrome (OMIM } \\
\# 113650)\end{array}$ & EYA1, SIX5 & $\begin{array}{l}\text { Ear anomalies (ear pits, microtia, anotia, branchial cysts), } \\
\text { polycystic kidneys, renal dysplasia, renal agenesis }\end{array}$ & $\mathrm{AD}$ \\
\hline $\begin{array}{l}\text { Renal-coloboma syndrome (OMIM } \\
\# 120330 \text { ) }\end{array}$ & $P A X 2$ & Fetal renal hypodysplasia, renal agenesis & $\mathrm{AD}$ \\
\hline $\begin{array}{l}\text { Renal cysts and diabetes syndrome } \\
\text { (OMIM \#137920) }\end{array}$ & $H N F 1 B$ & $\begin{array}{l}\text { Hyperparathyroidism, elevated liver enzymes, diabetes mellitus, } \\
\text { exocrine pancreatic dysfunction, hyperuricemia, urogenital } \\
\text { anomalies, renal cysts, impaired renal function, unilateral renal } \\
\text { agenesis }\end{array}$ & $\mathrm{AD}$ \\
\hline $\begin{array}{l}\text { Müllerian duct failure and } \\
\text { hyperandrogenism (OMIM \#158330) }\end{array}$ & WNT4 & $\begin{array}{l}\text { Aplasia of müllerian duct derivatives, hirsutism, rare unilateral } \\
\text { renal agenesis }\end{array}$ & $\mathrm{AD}$ \\
\hline $\begin{array}{l}\text { Miller-Dieker lissencephaly syndrome } \\
\text { (OMIM \#247200) }\end{array}$ & LIS1 & $\begin{array}{l}\text { Lissencephaly, midface hypoplasia, low set ears, thick upper lip, } \\
\text { omphalocele, renal agenesis }\end{array}$ & $\mathrm{AD}$ \\
\hline Goldenhar syndrome (OMIM \%164210) & SKI & $\begin{array}{l}\text { Craniosynostosis of coronal, sagittal, or lambdoid sutures, } \\
\text { hypertelorism, exophthalmos, micrognathia, marfanoid habitus, } \\
\text { developmental delay, renal agenesis }\end{array}$ & $\mathrm{AD}$ \\
\hline
\end{tabular}

Adapted from Talati et al., [2019]. XL, X-linked; AD, autosomal dominant; AR, autosomal recessive; CNV, copy number variant; VUR, vesicoureteral reflux. 
The input should be one or more phenotype terms, and the output is a list of candidate genes weighted by the chance of being associated with the phenotype. The result is based on the information contained within 5 disease-gene mapping databases: OMIM, ClinVar, GeneReviews, Orphanet, and GWAS catalog [Yang et al., 2015]. There are 2 different lists of genes provided as output by Phenolyzer: the seed gene list and the final gene list. The seed gene list includes genes having a confirmed relationship with the input phenotype, while the final gene list expands on genes hypothesized to be implicated in the disease pathogenesis based on their biological role and interactions only. Each time a gene is found to be directly associated with a disease, a weighted score is calculated, as previously described in Yang et al., 2015. To generate the Phenolyzer output, we submitted the following phenotype terms: absent kidney, renal agenesis, renal aplasia, unilateral renal agenesis, and bilateral renal agenesis. We decided to take into consideration the seed gene lists only and fused the lists obtained for each individual phenotype term into a single output list of genes.

We used the Cytoscape plugin ClueGO for the EA of this output list of genes. The outcome of the EA is a list of statistically enriched gene sets describing the biological properties common within the given gene list [Hall and Kiefer, 2016]. ClueGO is a software tool able to display the results of such an EA in the form of a network of interconnected biological processes, allowing for a more intuitive use and a clear interpretation of the results. There are several gene set libraries that can be used for EA such as Reactome, WikiPathways or the CORUM database of protein complexes [Kutmon et al., 2016; Fabregat et al., 2018; Giurgiu et al., 2019]. We selected Gene Ontology (GO; January 2020 version) due to the reliable content of its database, which provides structured and computable knowledge regarding the functions of genes and genes products, and the fact that it had already been used in other EA studies previously [Hall and Kiefer, 2016; Dalmer and Clugston, 2019]. The ontology covers 3 main aspects of gene function: molecular function (basic gene function at molecular level), cellular component (cellular location of the gene product), and biological process (series of molecular functions a gene is part of) [The Gene Ontology Consortium, 2019]. The output of EA through GO is a hierarchical ontology presented in a tabular form.

ClueGO is a Cytoscape plug-in that is able to integrate GO terms and transform them into a functionally organized network. We selected the Gene Ontology database of biological processes as a reference, in order to focus on altered developmental processes. Since GO has a hierarchical structure, the GO terms can be found at several levels, from very general (e.g., positive regulation of biological processes) to very specific terms (e.g., regulation of mesenchymal cell proliferation involved in ureter development). We selected the maximum specificity, in order to generate a reliable and meaningful network. This approach may, however, discard genes playing an unspecific role in universal pathways.

In GO, the $p$ value represents the chance of observing $n$ number of genes in a gene list annotated to a specific term [Dalmer and Clugston, 2019]. We used the standard corrected $p$ value $<0.05$ and kept the default ClueGO features (see online suppl. Fig. 1 for further details; see www.karger.com/doi/10.1159/000518115 for all suppl material). ClueGo statistical significance analysis is based on hypergeometric distribution test. To correct for multiple hypothesis testing, the program offers the choice between 3 different techniques: Bonferroni, Bonferroni step-down (better known as HolmBonferroni method), and Hochberg's step-up procedure. The de- fault is Holm-Bonferroni method, which we used for this study and we felt appropriate to include as part of this semi-automated procedure. Holm-Bonferroni is uniformly more powerful than standard Bonferroni and should therefore always be preferred unless simplicity and explainability are the focus. While the Hochberg procedure is uniformly more powerful than Holm-Bonferro$\mathrm{ni}$, it additionally requires the hypotheses to be independent (or at least non-negatively dependent), which cannot be guaranteed in all cases.

In the final output network, GO terms are grouped based on shared gene members. ClueGO uses kappa statistics to link the terms in the network. Similar GO terms are represented as nodes of the same color (i.e., circles), and the edges (i.e., the lines connecting the nodes) are the quantitative representation of shared gene membership (kappa score). The size of the nodes represents the enrichment significance of the terms [Bindea et al., 2009].

\section{Results}

Our seed genes list obtained using Phenolyzer contained 293 genes, 293 of which were associated to the "renal agenesis" phenotype term, 290 to "renal aplasia", 159 to the "absent kidney" query, 5 genes to "bilateral renal agenesis", and 45 to "unilateral renal agenesis". The list of genes for renal aplasia did not contain the genes SLC27A4, TBX6 and CTU2, which were included in the output gene list for renal agenesis. The output gene list for renal agenesis query included all genes that were found through the other searching attempts and included the 2 extra genes CTU2 and SLC27A4 which were not found in any of the other searches. Curating the list manually, 4 terms were eliminated since they corresponded to disease-associated chromosomal loci and not to genes (MPDMRS, DGCR, DER22T11-22, MGS), and 2 other genes were eliminated as they were synonyms (MCKD1 for MUC1, and $M A A$ for $N A A 10)$. The list of the 287 genes used for the EA is appended in the online supplementary material Table 1.

The gene EA identified 343 enriched GO terms with a standard $p<0.05$ (online suppl. Table 2 contains the complete list). The grouping of terms assigned them to 69 groups based on overlapping gene membership measured by kappa score statistics. The number of GO terms in the groups varied from a maximum of 100 to 29 groups with just 1 term each and 18 groups with shared GO terms. The output gene network is shown in Figure 1. Each node represents a GO term, while the edges (bundled in the picture for better display) are a quantitative representation of shared genes membership. Different colors of the nodes represent different groups generated. The name of the groups is automatically chosen by ClueGO, and it is the name of the most 


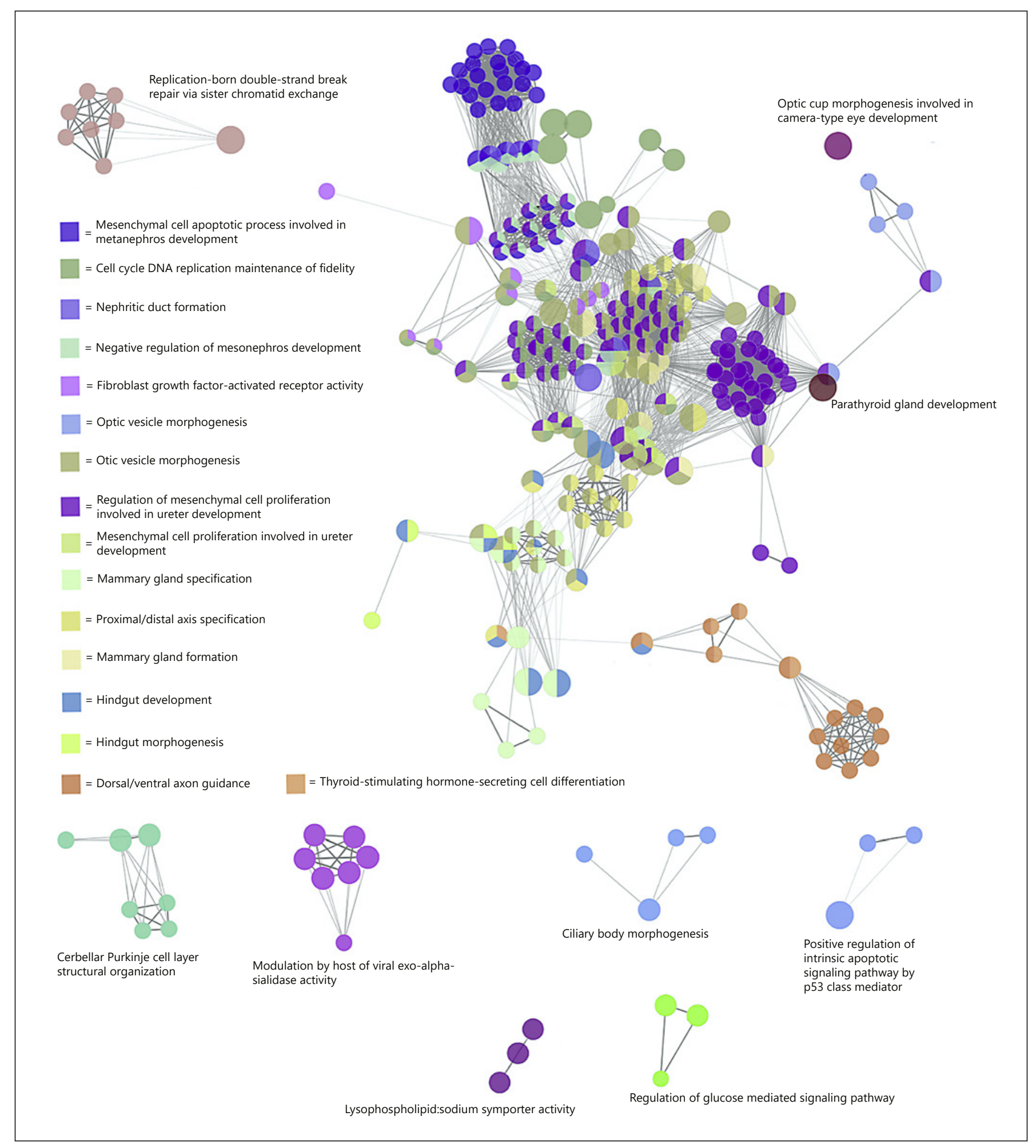

Fig. 1. Each node represents a gene ontology term, while the edges are a quantitative representation of shared genes membership. The different colors of the nodes represent the different groups generated. Only 24 groups out of 69 are represented in the figure, the ones shown are those that had an adjusted $p$ value lower than 0.05 . 
Table 2. 24 enriched gene ontology groups that had an adjusted $p$ value $<0.05$

\begin{tabular}{|c|c|c|c|c|}
\hline GO group & $\begin{array}{l}\text { GO terms within } \\
\text { the group }\end{array}$ & $\begin{array}{l}\text { No. of } \\
\text { genes }\end{array}$ & $\begin{array}{l}\text { Ajusted } \\
p \text { value }\end{array}$ & Gene names \\
\hline \multicolumn{5}{|l|}{ Biological process } \\
\hline Otic vesicle morphogenesis (GO:0071600) & 90 & 21 & $<0.0001$ & $\begin{array}{l}\text { BMP4, CDC42, DACT1, EYA1, FGF8, FGF10, FGFR1, FGFR2, FGFR3, GATA3, GLI1, GLI2, GLI3, HESX1, } \\
\text { HOXD13, NODAL, SHH, SIX1, TBX3, TP63, TNF }\end{array}$ \\
\hline $\begin{array}{l}\text { Cell cycle DNA replication maintenance of fidelity } \\
\text { (GO:1902298) }\end{array}$ & 35 & 13 & $<0.0001$ & BMP4, BRCA2, EYA1, FANCD2, FGFR1, FGFR2, GATA3, HMGA2, HNF1B, SHH, SIX1, RAD51, RFWD3 \\
\hline $\begin{array}{l}\text { Regulation of mesenchymal cell proliferation involved in } \\
\text { ureter development (GO:0072199) }\end{array}$ & 100 & 15 & $<0.0001$ & $\begin{array}{l}\text { BMP4, FGF10, FGFR1, FGFR2, GATA3, GLI3, HMGA2, HNF1B, PAX2, SHH, SIX1, SIX3, TBX3, TFAP2A, } \\
\text { TP63 }\end{array}$ \\
\hline Hindgut development (GO:0061525) & 12 & 10 & $<0.0001$ & DACT1, DCC, FGF8, GLI1, GLI2, GLI3, HOXD13, NODAL, SHH, SUFU \\
\hline Mammary gland specification (GO:0060594) & 16 & 9 & $<0.0001$ & DACT1, FGF10, FGFR2, GLI2, GLI3, HOXD13, SHH, STRA6, SUFU \\
\hline Mammary gland formation (GO:0060592) & 10 & 9 & $<0.0001$ & BMP4, FGF8, FGF10, FGFR2, GLI3, HESX1, SHH, TBX3, TNF \\
\hline Proximal/distal axis specification (GO:0009946) & 24 & 11 & $<0.0001$ & FGF8, FGF10, FGFR1, FGFR2, FGFR3, GLI1, GLI2, GLI3, HESX1, NODAL, SHH \\
\hline $\begin{array}{l}\text { Mesenchymal cell proliferation involved in ureter develop- } \\
\text { ment (GO:0072198) }\end{array}$ & 12 & 6 & $<0.0001$ & BMP4, EYA1, GATA3, HNF1B, SHH, SIX1 \\
\hline Nephritic duct formation (GO:0072179) & 12 & 7 & $<0.0001$ & BMP4, GATA3, HNF1B, PAX2, PTCH1, RET, SIX1 \\
\hline Hindgut morphogenesis (GO:0007442) & 4 & 5 & $<0.0001$ & DACT1, GLI2, GLI3, HOXD13, SHH \\
\hline $\begin{array}{l}\text { Negative regulation of mesonephros development } \\
\text { (GO:0061218) }\end{array}$ & 21 & 5 & $<0.0001$ & BMP4, GATA3, HNF1B, PAX2, SIX1 \\
\hline Parathyroid gland development (GO:0060017) & 1 & 4 & $<0.0001$ & CRKL, GATA3, PAX1, TBX1 \\
\hline $\begin{array}{l}\text { Optic cup morphogenesis involved in camera-type eye } \\
\text { development (GO:0002072) }\end{array}$ & 1 & 4 & $<0.0001$ & ARID1A, PAX2, SOX11, TFAP2A \\
\hline $\begin{array}{l}\text { Mesenchymal cell apoptotic process involved in metaneph- } \\
\text { ros development (GO:1900200) }\end{array}$ & 40 & 4 & $<0.001$ & BMP4, GATA3,HNF1B, PAX2 \\
\hline $\begin{array}{l}\text { Thyroid-stimulating hormone-secreting cell differentiation } \\
\text { (GO:0060129) }\end{array}$ & 6 & 4 & $<0.001$ & DCC, GLI2, FGF8, WNT4 \\
\hline $\begin{array}{l}\text { Positive regulation of intrinsic apoptotic signaling pathway } \\
\text { by p53 class mediator (GO:1902255) }\end{array}$ & 3 & 3 & $<0.001$ & RPL11, RPL26, RPS7 \\
\hline $\begin{array}{l}\text { Replication-born double-strand break repair via sister chro- } \\
\text { matid exchange (GO:1990414) }\end{array}$ & 8 & 3 & $<0.001$ & $F A N C B, N I P B L, R A D 51$ \\
\hline Optic vesicle morphogenesis (GO:0003404) & 5 & 4 & $<0.001$ & $B M P 4, S I X 3, T F A P 2 A$ \\
\hline $\begin{array}{l}\text { Fibroblast growth factor-activated receptor activity } \\
\text { (GO:0005007) }\end{array}$ & 9 & 3 & 0.0012 & FGFR1, FGFR2, FGFR3 \\
\hline Dorsal/ventral axon guidance (GO:0033563) & 14 & 3 & 0.0026 & DCC, FGF8, WNT4 \\
\hline $\begin{array}{l}\text { Regulation of glucose mediated signaling pathway } \\
\text { (GO:1902659) }\end{array}$ & 3 & 2 & 0.0057 & SMARCA4, SMARCB1 \\
\hline $\begin{array}{l}\text { Lysophospholipid: Sodium symporter activity } \\
\text { (GO:0051978) }\end{array}$ & 3 & 2 & 0.0057 & MFSD2A, PHGDH \\
\hline $\begin{array}{l}\text { Cerebellar Purkinje cell layer structural organization } \\
\text { (GO:0021693) }\end{array}$ & 7 & 2 & 0.0099 & DLL1, KIF14 \\
\hline $\begin{array}{l}\text { Modulation by host of viral exo-alpha-sialidase activity } \\
\text { (GO:0044866) }\end{array}$ & 7 & 2 & 0.0099 & PITX2, РITX 3 \\
\hline Ciliary body morphogenesis (GO:0061073) & 4 & 2 & 0.021 & JAG1, NOTCH2 \\
\hline
\end{tabular}

The complete list of groups and gene ontology (GO) terms are present in online supplementary Table 2. Next to each GO group is the number of GO terms contained within the groups, the number of genes that contributed to each group enrichment, and the list of such genes. The groups are ranked according to their $p$ value.

relevant GO term within the group. When groups overlap, the next most significant term is used (see bold GO terms in online suppl. Table 2). Some biological pro- cesses (i.e., GO terms) are present in more than one group, hence some of the nodes are pie-chart-like, with different colors representing the groups' membership. 


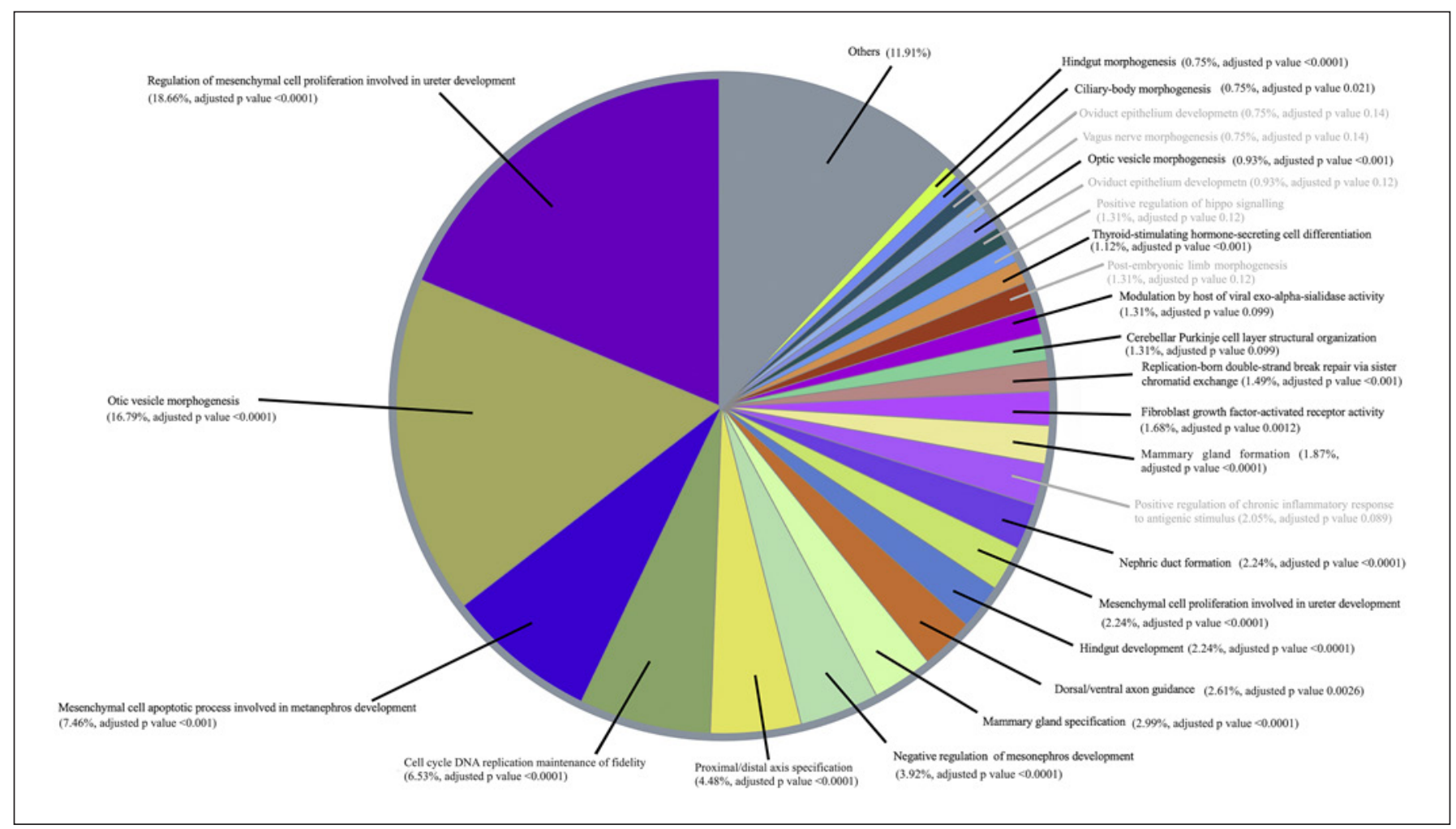

Fig. 2. Pie chart representing ClueGo pathway groups according to the percentage of gene ontology (GO) terms contained with respect to the total (536, considering the overlaps). Only 26 groups out of 69 are represented in the chart, groups containing less than $0.75 \%$ of the total GO terms are represented in gray, and all to-

In Figure 1, only the 24 groups whose adjusted $p$ value $<0.05$ are represented, such groups are better described in Table 2, where they are ranked according to their $p$ value (the complete list of groups and GO terms are present in online suppl. Table 2). Figure 2 depicts the groups according to the percentage of GO terms contained with respect to the total number of terms (536, considering the overlaps).

\section{Discussion}

Renal agenesis, particularly when bilateral, has a poor prognosis with little to no chance of survival, and is considered the severe end of CAKUT, accounting for up to $20 \%$ of the congenital anomalies. The demand to identify the etiology is high since it would inform pregnancy management, prognosis, and recurrence risk. There is increasing evidence that monogenic causes are playing a prominent role, even in isolated forms of renal agenesis. gether they represented $11.91 \%$ of all the GO terms (see online suppl. Table 2 for the complete list). Next to each group name is the relative adjusted $p$ value, name groups that had a $p$ value $>0.05$ are written in light gray.

However, recognized clinical and genetic heterogeneity and diversity present a challenge in clinical and diagnostic approaches, including the interpretation of genomewide sequencing data. There is a clear need to functionally group the genes involved into known pathways and biological processes in order to provide structured and meaningful analyses, and to use this potential to identify and prioritize novel candidate genes and pathways.

Important bioinformatic tools are being developed in order to enable also non-bioinformaticians to perform the necessary data analysis. We took advantage of Phenolyzer to create a list of genes associated with renal agenesis, allowing for a standardized, reproducible, and exhaustive search as compared to a manual literature search (e.g., Medline, PubMed and OMIM), which is classically used to obtain a list of genes associated with a congenital anomaly [Hall and Kiefer, 2016; Kiefer and Hall, 2019]. Through this approach, we were able to extract 287 genes that are associated with the term "renal agenesis", either isolated or syndromic. The phenotype term "renal agenesis" allowed to generate the 
most comprehensive list of genes, exceeding all of the other terms used (i.e., "absent kidney", "renal aplasia", "unilateral renal agenesis", "bilateral renal agenesis"), and it cannot be obtained by simply combining the gene output list for BRA and URA. The terms "renal aplasia" and "absent kidney" failed to generate the same output, despite being synonyms. A possible explanation is the word-based literature search algorithm of Phenolyzer and the preferred use of the term "agenesis" in recent publications. We therefore suggest to use the broader and more commonly used phenotype terms for Phenolyzer inputs in order to generate the most comprehensive outputs. CTU2 (OMIM *617057) and SLC27A4 (OMIM *604194) gene associations were only obtained when searching for renal agenesis and failed to be identified through any other search. While variants in CTU2 are associated with microcephaly, facial dysmorphism, renal agenesis, and ambiguous genitalia syndrome according to the OMIM database (OMIM \#618142), a manual search through the literature failed to show a direct association between the SLC27A4 gene and renal agenesis. Yet pathogenic variants in the SLC27A4 gene can cause the ichthyosis prematurity syndrome (OMIM \#608649) [Krishnamurthy et al., 2007], illustrated by a neonate reported with this type of ichthyosis, as well as Kallmann syndrome and URA. Such false-positive calls, however, seem to be very rare ( 1 out of 287 genes) and may be produced by text mining algorithms leading to the inclusion of genes which are not relevant for the phenotype term. On 2 occasions, Phenolyzer outputted genes twice because of their existing alternative names (i.e., MCKD1/MUC1 and MAA/NAA10). We also identified calls indicative of chromosomal loci (e.g., DGCR, i.e., Di George Critical Region) associated with renal agenesis. Future algorithms may be improved to correct for such calls and may provide information on chromosomal loci separately from the gene list for monogenic disorders. At present, despite the efficacy of Phenolyzer, a manual curation of the output list is still important to improve its accuracy. As machine learning techniques are constantly improving, we expect the bioinformatic algorithms of such tools to be increasingly reliable, efficient, and sophisticated. They may even become useful in the daily clinical and molecular genetics practice, whenever they are amenable into a user-friendly interface for non-bioinformaticians.

As expected, the EA of the curated output list of genes reflects the huge heterogeneity of the genetic and biological pathways underlying renal agenesis. Not surprisingly, pathways directly involved in kidney development, such as regulation of mesenchymal cell proliferation involved in ureter development (adjusted $p$ value $<0.0001$ ), nephric duct for- mation (adjusted $p$ value $<0.0001$ ), negative regulation of mesonephros development (adjusted $p$ value $<0.0001$ ), mesenchymal cell apoptotic process involved in metanephros development (adjusted $p$ value $<0.0001$ ), proximal/distal axis specification (adjusted $p$ value $<0.0001$ ), and cell cycle regulation (cell cycle DNA replication maintenance of fidelity, adjusted $p$ value $<0.0001$; positive regulation of intrinsic apoptotic signaling pathway by $\mathrm{p} 53$ class mediator, adjusted $p$ value $<0.001$; replication-born double-strand break repair via sister chromatid exchange, adjusted $p$ value $<0.001$ ), contribute in a significant proportion. This is in part probably biased by the fact that genes involved in kidney development were primarily identified and studied in the context of renal agenesis. A number of pathways involved in the development of the hindgut (hindgut development and hindgut morphogenesis, adjusted $p$ value for both $<0.0001$ ), parathyroid glands (adjusted $p$ value $<0.0001$ ), optic morphogenesis (optic cup morphogenesis involved in camera-type eye development, adjusted $p$ value $<0.0001$; optic vesicle morphogenesis, adjusted $p$ value $<0.001$ ), and neural development (dorsal/ventral axon guidance, adjusted $p$ value 0.0026; cerebellar Purkinje cell layer structural organization, adjusted $p$ value 0.0099 ) may explain the associated developmental field defects, representing a particular etiologic group with an important impact on phenotype expression. Other pathways such as the mammary gland specification/formation ( $p$ value $<0.0001$ for both) and thyroid cells differentiation (thyroid-stimulating hormone-secreting cell differentiation, adjusted $p$ value $<0.001$ ) are more surprising to find, but suggest shared developmental processes. Such findings present the opportunity to identify many more genes which, when altered, are involved in kidney development and its anomalies.

Interestingly, ciliary body morphogenesis (adjusted $p$ value 0.021 ) is a pathway represented in a very small proportion. The first biological process group ranked according to $p$ value is otic vesicle morphogenesis (adjusted $p$ value $<0.0001$ ), a structure of the neural ectoderm that later develops into the membranous labyrinth of the inner ear. In zebrafish models, the otic vesicle development is key to evaluate the presence or absence of a ciliopathy, as motile and immotile cilia are of key importance for the development of this structure [Stooke-Vaughan et al., 2012; Song et al., 2016]. A debate has indeed been recently ongoing whether a ciliopathy must be clinically considered when renal agenesis is present in an otherwise suggestive syndromic phenotype [Devlin and Sayer, 2019] or even when isolated. Renal ciliopathies are usually characterized by cystic dysplastic kidney disease or nephronophthisis, whose underlying pathogenesis is related to abnormal 
structure or function of the primary cilium [Devlin and Sayer, 2019]. Renal hypodysplasia has not traditionally been linked to ciliopathic renal disease, even less so in the case of renal agenesis [Waters and Beales, 2011]. Nevertheless, URA or BRA have recently been described in association with ciliopathies. Two prenatally identified cases of Meckel-Gruber syndrome were reported in association with URA [Nyberg et al., 1990; Uysal and Uysal, 2015]. Filges et al., 2013 reported BRA in a fetus affected by an autosomal recessive ciliopathy (Meckel syndrome type 12; OMIM \#616258), while phenotype recurrence in the same family presented with dysplastic kidneys. Grampa et al., 2016 described a fetus with URA and contralateral renal dysplasia, as well as cardiomegaly, paucity of bile ducts, short and fibrotic pancreas, asplenia, narrow thorax and short and bowed legs, carrying heterozygous variants in the NEK8 gene, whose encoded protein localizes within the inversin compartment of the cilium. Most recently Quélin et al. [2018] reported a 18-week-old fetus having BRA, short ribs, polydactyly and imperforate anus, carrying heterozygous loss-of-function variants in the IFT27 gene, coding for a component of the cilia intraflagellar transport complex. The results of the EA support the hypothesis that renal agenesis is likely part of the ciliopathy spectrum and developmentally related to dysplastic kidneys.

The purpose of this paper was to provide a logically structured approach by highlighting the genes in which variants have been identified to be associated with renal agenesis and to define the pathways involved in this type of abnormal kidney development. The output gene list we provide (see online suppl. Table 1) can be used to develop a comprehensive diagnostic approach, and our overall approach can be transferred to the investigation of developmental principles of other congenital anomalies which are usually heterogeneous in etiology. In the future, the diagnostic detection rates of such gene output lists need to be validated in prospective prenatal cohorts of patients.

Besides some inaccuracies encountered in the use of Phenolyzer, there are few limitations to our input data for EA, particularly when thinking about the delineation of novel candidate genes and pathways. We restricted the gene list further used for EA to the seed genes having a confirmed relationship with the input phenotype. This could potentially be expanded on genes which are only hypothesized to be implicated in the disease pathogenesis due to their biological role and interactions (the so-called "final gene list" in Phenolyzer), which would be prohibitive for a diagnostic approach, but useful in a research setting. Another limitation is inherent to the Gene Ontology database, since a function of a gene can be very com- plex to determine and may be strongly dependent on the context. It is indeed difficult to distinguish between the properties which are intrinsic to the gene per se and those which depend on the contextual factors (e.g., different cell types, tissues or organs or different stages of the same cell lineage or interacting proteins). Furthermore, each gene can give rise to many different proteins, depending on its post-translational modifications. The attribution of function also largely depends on the type and scenarios of experiments used to derive it [Greenspan, 2011]. In addition, multifactorial, epigenetic, and environmental factors may cause or influence the etiology and/or clinical manifestation of an anomaly.

The study of genes in which variants have been identified to be associated with renal agenesis using GO and EA highlights the importance of a structured logical approach for diagnosis as well as the understanding of the diverse developmental processes involved. This might help to reach an early diagnosis of the causal genetic disorder, providing the means to conceive appropriate gene panels and interpret genome-wide sequencing data, thus finally improving the clinical management and counseling for prognosis and recurrence risk. The exercise is also useful for a comprehensive discussion of the pathogenesis of the disease, as well as for shedding light on novel candidate genes and developmental pathways that might be discovered in the future in association with the kidney anomaly, and ultimately the research for new targeted therapies. Importantly, the bioinformatic tools we applied can be used by non-bioinformaticians. We believe it is crucial that in the era of high-throughput genomic diagnostic and experimental data, clinical geneticists familiarize with bioinformatic tools which use artificial intelligence to structure our understanding of the phenotype-genotype-pathway relationships, ultimately contributing to a better use of its unprecedented power for the benefit of our patients.

\section{Acknowledgement}

We gratefully acknowledge the support of Claudio Peroni, data scientist, who counseled us with regards to the technical aspects of the paper.

\section{Statement of Ethics}

An ethics statement was not required for this study type, no human or animal subjects or materials were used. 


\section{Conflict of Interest Statement}

The authors declare that the research was conducted in the absence of any commercial or financial relationships that could be construed as a potential conflict of interest.

\section{Funding Sources}

The Swiss National Science Foundation (SNSF), Project Grant to Isabel Filges (320030_160200) supported this work.

\section{Author Contributions}

S.K. and I.F. both developed the concept and wrote the manuscript.

\section{Data Availability Statement}

All data generated or analyzed during this study are included in this article and its supplementary material files. Further enquiries can be directed to the corresponding author.

\section{References}

Bianchi DW, Combleholme TM, D'Alton ME Malone FD. Fetology. McGraw-Hill Education/Medical; 2010. p. 1024.

Bindea G, Mlecnik B, Hackl H, Charoentong P, Tosolini M, Kirilovsky A, et al. ClueGO: A Cytoscape plug-in to decipher functionally grouped gene ontology and pathway annotation networks. Bioinformatics. 2009;25(8): 1091-3.

Bodria M, Sanna-Cherchi S. Genetic Basis of Congenital Anomalies of the Kidney and Urinary Tract (in Italian). G Ital Nefrol. 2015;32 Suppl 64:gin/32.S64.16.

Dalmer TRA, Clugston RD. Gene ontology enrichment analysis of congenital diaphragmatic hernia-associated genes. Pediatr Res. 2019; 85(1):13-9.

Devlin LA, Sayer JA. Renal ciliopathies. Curr Opin Genet Dev. 2019;56:49-60.

Fabregat A, Korninger F, Viteri G, Sidiropoulos K, Marin-Garcia P, Ping P, et al. Reactome graph database: Efficient access to complex pathway data. PLoS Comput Biol. 2018;14(1): e1005968.

Filges I, Nosova E, Bruder E, Tercanli S, Townsend $\mathrm{K}$, Gibson WT, et al. Exome sequencing identifies mutations in KIF14 as a novel cause of an autosomal recessive lethal fetal ciliopathy phenotype. Clin Genet. 2014;86(3):220-8.

Giurgiu M, Reinhard J, Brauner B, DungerKaltenbach I, Fobo G, Frishman G, et al. CORUM: The comprehensive resource of mammalian protein complexes - 2019. Nucleic Acids Res. 2019;47(D1):D559-D563.

Grampa V, Delous M, Zaidan M, Odye G, Thomas S, Elkhartoufi N, et al. Novel NEK8 Mutations Cause Severe Syndromic Renal Cystic Dysplasia through YAP Dysregulation. PLoS Genet. 2016;12(3):e1005894.

Greenspan NS. Attributing functions to genes and gene products. Trends Biochem Sci. 2011; 36(6):293-7.

Groopman EE, Marasa M, Cameron-Christie S, Petrovski S, Aggarwal VS, Milo-Rasouly H, et al. Diagnostic Utility of exome sequencing for kidney disease. N Engl J Med. 2019;380(2): $142-51$.
Hall JG, Kiefer J. Arthrogryposis as a Syndrome: Gene Ontology Analysis. Mol Syndromol. 2016;7(3):101-9.

Harewood L, Liu M, Keeling J, Howatson A, Whiteford M, Branney P, et al. Bilateral renal agenesis/Hypoplasia/Dysplasia (BRAHD): Postmortem analysis of 45 cases with breakpoint mapping of two De novo translocations. PLoS One. 2010;5(8):e12375.

Jin M, Zhu S, Hu P, Liu D, Li Q, Li Z, et al. Genomic and epigenomic analyses of monozygotic twins discordant for congenital renal agenesis. Am J Kidney Dis. 2014;64(1):11922.

Kiefer J, Hall JG. Gene ontology analysis of arthrogryposis (multiple congenital contractures). Am J Med Genet C Semin Med Genet. 2019;181(3):310-26

Kleiner B, Filly RA, Mack L, Callen PW. Multicystic dysplastic kidney: Observations of contralateral disease in the fetal population. Radiology. 1986;161(1):27-9.

Krishnamurthy S, Kapoor S, Yadav S. Case Reports Nephrotic Syndrome with X-Linked Ichthyosis, Kallmann Syndrome and unilateral renal agenesis. Indian Pediatr.2007;44(4): 301-3.

Kutmon M, Riutta A, Nunes N, Hanspers K, Willighagen EL, Bohler A, et al. WikiPathways: Capturing the full diversity of pathway knowledge. Nucleic Acids Res. 2016;44(D1):D48894.

Mesrobian HG, Rushton HG, Bulas D. Unilateral renal agenesis may result from in utero regression of multicystic renal dysplasia. J Urol. 1993;150(2 Pt 2):793-4.

Nyberg DA, Hallesy D, Mahony BS, Hirsch JH, Luthy DA, Hickok D. Meckel-Gruber syndrome. Importance of prenatal diagnosis. J Ultrasound Med. 1990;9(12):691-6.

Quélin C, Loget P, Boutaud L, Elkhartoufi N, Milon J, Odent S, et al. Loss of function IFT27 variants associated with an unclassified lethal fetal ciliopathy with renal agenesis. Am J Med Genet A. 2018;176(7):1610-3.
Rossi AC, Prefumo F. Accuracy of ultrasonography at 11-14 weeks of gestation for detection of fetal structural anomalies: A systematic review. Obstet Gynecol. 2013;122(6):1160-7.

Sanna-Cherchi S, Caridi G, Weng PL, Scolari F, Perfumo F, Gharavi AG, et al. Genetic approaches to human renal agenesis/hypoplasia and dysplasia. Pediatr Nephrol. 2007;22(10): 1675-84.

Song Z, Zhang X, Jia S, Yelick PC, Zhao C. Zebrafish as a Model for Human Ciliopathies. J Genet Genomics. 2016;43(3):107-20.

Stooke-Vaughan GA, Huang P, Hammond KL, Schier AF, Whitfield TT. The role of hair cells, cilia and ciliary motility in otolith formation in the zebrafish otic vesicle. Development. 2012;139(10): 1777-87.

Talati AN, Webster CM, Vora NL. Prenatal genetic considerations of congenital anomalies of the kidney and urinary tract (CAKUT). Prenat Diagn. 2019;39(9):679-92.

The Gene Ontology Consortium . The Gene Ontology Resource: 20 years and still GOing strong. Nucleic Acids Res. 2019;47(D1): D330-8.

Uysal F, Uysal A. Meckel-Gruber syndrome with unilateral renal agenesis. J Coll Physicians Surg Pakistan. 2015;25:S56-7.

Verbitsky M, Westland R, Perez A, Kiryluk K, Liu $Q$, Krithivasan $P$, et al. The copy number variation landscape of congenital anomalies of the kidney and urinary tract. Nat Genet. 2019; 51(1):117-27.

Waters AM, Beales PL. Ciliopathies: An expanding disease spectrum. Pediatr Nephrol. 2011; 26(7):1039-56.

Wu H, Xu Q, Xie J, Ma J, Qiao P, Zhang W, et al. Identification of 8 Novel Mutations in Nephrogenesis-Related Genes in Chinese Han Patients with Unilateral Renal Agenesis. Am J Nephrol. 2017;46(1):55-63.

Yang H, Robinson PN, Wang K. Phenolyzer: Phenotype-based prioritization of candidate genes for human diseases. Nat Methods. 2015; 12(9):841-3. 